\title{
PATRÓN DE ACTIVACIÓN HEMISFÉRICA EN ADULTOS EN TAREAS DE LECTURA DE PALABRAS Y CONCIENCIA FONOLÓGICA MEDIDAS CON UNA NUEVA METODOLOGÍA DE MEDIDA DE LA ACTIVIDAD ELECTRODERMAL DIFERENCIAL (EDA-D)
}

\author{
Cristian García Zazo \\ SIADOE, EU de Educación y Turismo \\ Universidad de Salamanca, Ávila, España \\ cristiangz@usal.es \\ M. Isabel Valdunquillo Carlón \\ Departamento de Psicología Evolutiva y de la Educación \\ EU de Educación y Turismo \\ Universidad de Salamanca, Ávila, España \\ José Luis Martínez Herrador \\ Departamento de Psicología Evolutiva y de la Educación \\ EU de Educación y Turismo \\ Universidad de Salamanca, Ávila, España
}

Fecha de Recepción: 7 Enero 2019

Fecha de Admisión: 30 Abril 2019

\section{RESUMEN}

Aunque los procesos lectores están bien documentados desde modelos cognitivos, se conoce menos su correlato neurofuncional y lateralización. Está estudiada la participación hemisférica en lenguaje oral y en algunos procesos del lenguaje escrito, localizados preferentemente en el hemisferio izquierdo, pero no se ha descrito la existencia de un patrón de activación diferenciado en tareas de lectura de palabras y conciencia fonológica.

Para analizar estas variables desde esta perspectiva, se presenta una nueva instrumentación tecnológica y metodología de análisis para registrar la actividad electrodermal (EDA) y la actividad electrodermal diferencial (EDA-D) como indicador de la asimetría hemisférica. El instrumento mide el valor diferencial de la variable EDA y ha sido utilizado en otras aplicaciones, permitiendo conocer la activación hemisférica preferente ante tareas. Se registró la activación hemisférica diferencial mediante EDA en tareas de lectura de palabras y conciencia fonológica en 30 participantes (15 mujeres y 15 varones). Se registró también la activación basal de cada participante como medida inicial; posteriormente, se presentaron en formato powerpoint, cuatro tareas de lectura: palabras en euskera, palabras suecas, pseudopalabras y palabras familiares, y dos tareas de conciencia fonológica. Los resultados muestran patrones diferenciados en y entre algunas tareas.

Palabras clave: asimetría hemisférica; actividad electrodermal; lectura; conciencia fonológica 


\title{
PATRÓN DE ACTIVACIÓN HEMISFÉRICA EN ADULTOS EN TAREAS DE LECTURA DE PALABRAS Y CONCIENCIA FONOLÓGICA MEDIDAS CON UNA NUEVA METODOLOGíA DE MEDIDA DE LA ACTIVIDAD ELECTRODERMAL DIFERENCIAL (EDA-D)
}

\begin{abstract}
Pattern of hemispheric activity in adults in work reading tasks and phonological awareness measures with a new methodology of measurement of differential electrodermal activity (edad). Although reading is well studied from cognitive models, less is known about its neurofunctional correlate and lateralization. Hemispheric participation in oral language and in some written language processes, preferably located in the left hemisphere, is documented, but it is not yet known whether there is a differentiated activation pattern in tasks such as word reading and phonological awareness.

To analyze these variables from this perspective, a new technological instrumentation and analysis methodology is presented to record electrodermal activity (EDA) and differential electrodermal activity (EDA-D) as an indicator of hemispheric asymmetry. The instrument measures the differential value of the EDA variable and has been used in other applications, allowing to know the preferential hemispheric activation before tasks. The differential hemispheric activation by means of EDA was recorded in word reading and phonological awareness tasks in 30 participants (15 women and 15 men). Each participant's basal hemispheric activation was also recorded; later, four reading tasks were presented in powerpoint format: words in Basque, Swedish words; pseudo- words and familiar words, and two phonological awareness tasks. Results show differentiated patterns in and between some tasks
\end{abstract}

Keywords: hemispheric asymmetry; electrodermal activity; reading; phonological awareness

\section{ANTECEDENTES}

Distintos estudios refieren una participación hemisférica diferenciada en procesos básicos del lenguaje oral y escrito. Existen evidencias (Cuetos, 2018; Dehaene, 2007, 2018; López-Escribano, 2009) de que el hemisferio izquierdo interviene en la programación motora de los sonidos, sintaxis, parte de la semántica, procesos visuales ortográficos y correspondencia fonema-grafema. Otros muestran que el hemisferio derecho participa en el reconocimiento de letras y palabras concretas, acceso al significado, prosodia y comprensión contextual (Ardila, Bernal y Rosselli, 2016; Cantú, Lera y Baca, 2017; Monsalve y Cuetos, 2001). A pesar de estas evidencias no se ha documentado aún la existencia de una activación hemisférica diferencial en procesos más específicos en lenguaje escrito, en concreto, en lectura de palabras y conciencia fonológica.

En lectura de palabras, los modelos cognitivos refieren dos vías de acceso al léxico: la fonológica para palabras desconocidas y pseudopalabras, mediante el principio alfabético, y la léxica, con acceso directo (Alegría, Carrillo y Sánchez, 2005; Colheart, 1978; Defior, Serrano y Gutiérrez, 2015; Rueda, 2017). Ambas tienen un correlato neurofuncional, la palabra es procesada en el área occipito-temporal, nombrándose en el área de Broca, excepto con pseudopalabras, decodificadas en el área de Wernicke, como paso intermedio (Dehaene, 2018). Las tareas de conciencia fonológica se procesan en el hemisferio izquierdo (Mejía y Eslava, 2008).

Son numerosas las pruebas utilizadas para evaluar lectura de palabras (Test para la detección de la Dislexia en Niños: DST-J (Fawcett y Nicholson, 2013), Batería de Evaluación de los Procesos Lectores, Revisada PROLEC-R (Cuetos, Rodríguez, Ruano y Arribas, 2007) y conciencia fonológica (Prueba para la Evaluación del Conocimiento Fonológico, PECO (Ramos y Cuadrado, 2006). Desde la neurociencia, las técnicas de neuroimagen han aportado también evidencias (Gerrits, Van der Haegen, Brysbaerty y Vingerhoets, 2019; Porta, Kraft y Harper, 2009; Roessman et al., 2019). La actividad electrodermal (EDA) y la actividad electrodermal diferencial (EDA-D), técnica psicofisiológica indirecta de la activación hemisférica (Boucsein, 2012; Hellerud, 2002; Mojica-Londoño, 2017; Moncada y De la Cruz, 2011; Rippon, 1993), y documentada como indicador de procesos cognitivos (Roessman et al., 2019). 


\section{OBJETIVOS}

Este trabajo pretende comprobar si el instrumento desarrollado permite, mediante el registro de variables electrodérmicas, identificar patrones de activación hemisférica en tareas de lectura de palabras y conciencia fonológica.

\section{PARTICIPANTES}

La muestra se configuró con 30 participantes, adultos universitarios (15 mujeres y 15 varones) $(=24,5 ; \mathrm{SD}=4,14)$.

\section{MÉTODO}

\section{Instrumentos y variables}

Se diseñó una instrumentación tecnológica para registrar la actividad electrodermal diferencial (EDA-D) como indicador de la asimetría hemisférica. El instrumento fue un prototipo de Sociograph desarrollado por el equipo y ya utilizado (Martínez y Valdunquillo, 1983; Martínez, Garrido y Valdunquillo, 2005 Martínez, Monge y Valdunquillo, 2012) (Figura 1). Su diseño y desarrollo se realizó a partir de tecnologías ya conocidas (Boucsein, 2012), con nuevos procedimientos de análisis para separar las señales EDL de la NSA y EDR. El sistema de medición fue el de Corriente Constante (CC), medida en Kilohmios (K $\Omega$ ).

Figura 1. Instrumento de medición de EDA y EDA-D utilizado.

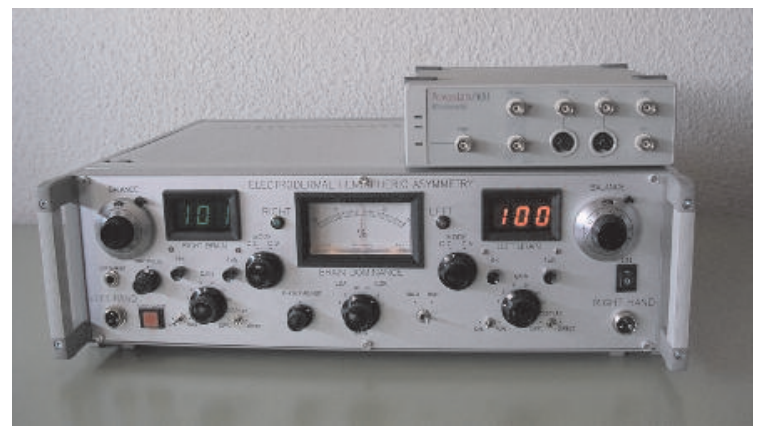

Los datos analógicos fueron digitalizados mediante el sistema de adquisición de datos ADinstruments PowerLab 425, procesados con LabChart 6, y analizados con SPSS 20. Los registros se efectuaron con un muestreo de 100 muestras por segundo para garantizar una alta precisión en tiempos de respuesta y amplitud y para evaluar elementos que no se han considerado en la mayoría de mediciones de la EDA. Cada canal registró 6600 datos por tarea, ello permitió medir con gran precisión diferencias de tiempos de reacción ante estímulos o latencias entre hemisferios y duración de las respuestas. Las variables básicas registradas quedan reflejadas en la Tabla 1. 


\section{PATRÓN DE ACTIVACIÓN HEMISFÉRICA EN ADULTOS EN TAREAS DE LECTURA DE PALABRAS Y CONCIENCIA FONOLÓGICA MEDIDAS CON UNA NUEVA METODOLOGíA DE MEDIDA DE LA ACTIVIDAD ELECTRODERMAL DIFERENCIAL (EDA-D)}

Tabla 1. Variables básicas registradas

\begin{tabular}{ll} 
1. EDL-HD & HD \\
\hline 2. EDL-HI & HI \\
\hline 3. EDR-HD & HD \\
\hline 4. EDR-HI & HD \\
\hline 5. Coeficiente de asimetría & \\
\hline
\end{tabular}

EDL: Electrodermal activity level; EDR: Electrodermal activiy response; HD: Hemisferio derecho; HI: Hemisferio izquierdo.

Para calcular la variable 5 o Coeficiente de Asimetría (Román-Lapuente, García-Sánchez y López-Amor, 1986) se aplicó la fórmula de diferencias proporcionales de la actividad EDL:

$$
\frac{(E D L . H D-E D L . H I)}{(E D L . H D+E D L . H I)}
$$

Esta ecuación supone un índice de proporcionalidad mostrando cuándo la activación de un hemisferio es igual 0 superior al otro (valores +1 y -1 ). Los positivos indican activación hemisférica izquierda y los negativos, derecha (Freixa y Baqué, 1984). El Coeficiente de Asimetría indica en qué proporción un hemisferio está más activado que el otro. Esta variable es específica de cada persona pero, a su vez, puede verse modificada al ser expuesta a cada tarea, tal como sucede en este estudio en el que se presentan diferentes tareas para comprobar cambios en la activación hemisférica. Si los cambios producidos se manifestaran como activación hemisférica derecha, tenderían hacia valores negativos y, en caso contrario, a una mayor activación izquierda.

El sistema de recogida y análisis de datos permitió evaluar otras variables cuantitativas y transformadas relevantes. La variable Pendiente o Slope, muestra la magnitud, dirección y velocidad del cambio en la EDL como reacción ante un estímulo. Los cambios se refieren a incrementos o decrementos de la resistencia. Si el valor de la pendiente es positivo indica aumento de resistencia, o disminución de la activación; en caso contrario, muestra disminución de resistencia, 0 aumento de activación. La variable Integral bajo mínimos permite cuantificar el valor de una respuesta y determinar su significación, permitiendo alcanzar mayores niveles de precisión que con otros métodos más utilizados en la medición de la variable EDR, puesto que una respuesta puede tener una amplitud mayor que otra (leptocúrtica) pero menor duración, o una amplitud menor pero mayor duración (platicúrtica).

\section{PROCEDIMIENTO}

Se colocó a cada participante dos electrodos de plata de $1 \mathrm{~cm}$ cuadrado de superficie, en la segunda falange de los dedos índice y corazón de cada mano. Se les proporcionó la misma consigna por la misma persona antes de la tarea. Se tomó una medida basal a cada uno durante un minuto; tras ello, se presentaron las tareas en PowerPoint. Se proyectaron los estímulos de cada tarea con tiempos de exposición predeterminados y separados por una transición en la que figuraba un impulso incluido en cada registro, para controlar los tiempos de respuesta en cada estímulo automáticamente. Ello permitió medir los tiempos de respuesta ante cada estímulo (en función de su dificultad), o diferencias de respuesta en la activación hemisférica. Entre cada tarea se presentaron estímulos de carácter neutro, evitando un efecto arrastre. Las tareas presentadas configuraron dos estudios diferentes, valorados independientemente. 
Esta técnica aporta numerosos datos que requieren análisis complejos aún no explorados. Por ello, solo se presentan los resultados de las tareas y su relación entre ellas, quedando pendiente el análisis dentro de cada tarea, así como las respuestas a cada tipo de estímulo.

\section{Estudio 1}

Se presentaron cuatro tareas: 1) 10 palabras en euskera (Ferrero y Goikoetxea, 2007); 2) 10 palabras suecas; 3) 10 pseudopalabras; y 4) 10 palabras familiares de alta frecuencia (Fajardo, Hernández y González, 2012), leídas y producidas oralmente.

\section{Estudio 2}

Se presentaron dos tareas de conciencia fonológica: 5) 10 palabras para eliminar el primer sonido y producir la palabra resultante; 6) 10 palabras para indicar el tercer sonido. En ambas se registraron aciertos y errores de los participantes.

\section{RESULTADOS}

Se procedió al tratamiento de los datos de cada una de las variables y fragmentos. En primer lugar, se presenta, como ejemplo un análisis visual de los registros de uno de los participantes, para poder observar y comprender mejor su análisis. En segundo lugar, se presentan los datos cuantitativos de cada estudio individualmente.

Análisis cualitativo de los registros gráficos

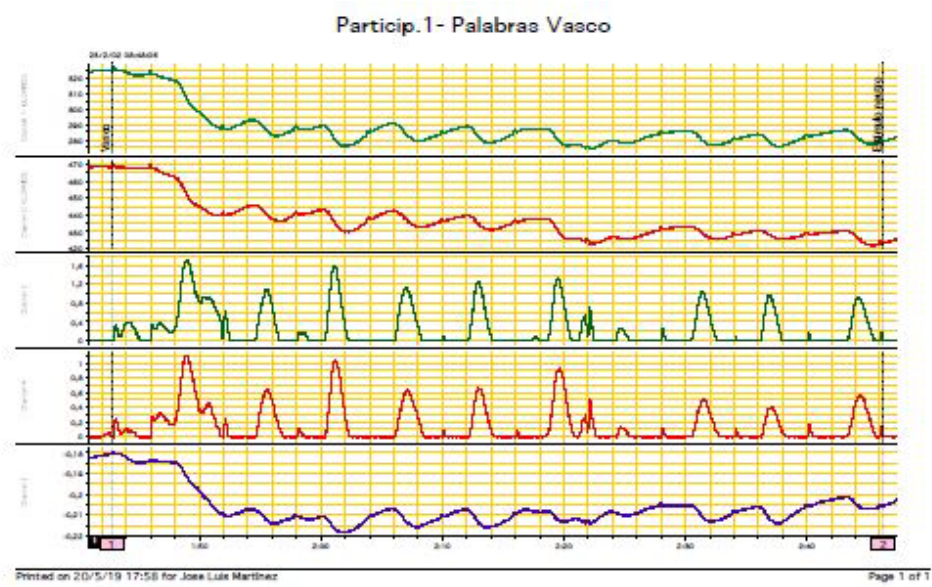

Figura 2. Registro gráfico de la tarea 1 (palabras en euskera) del participante 1 


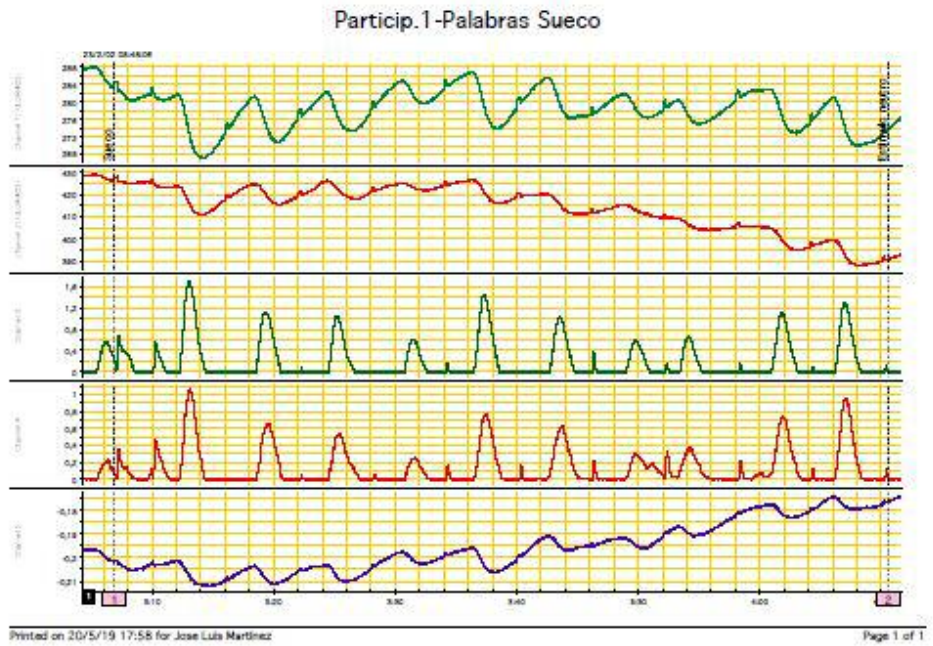

Figura 3. Registro gráfico de la tarea 2 (palabras en euskera) del participante 1

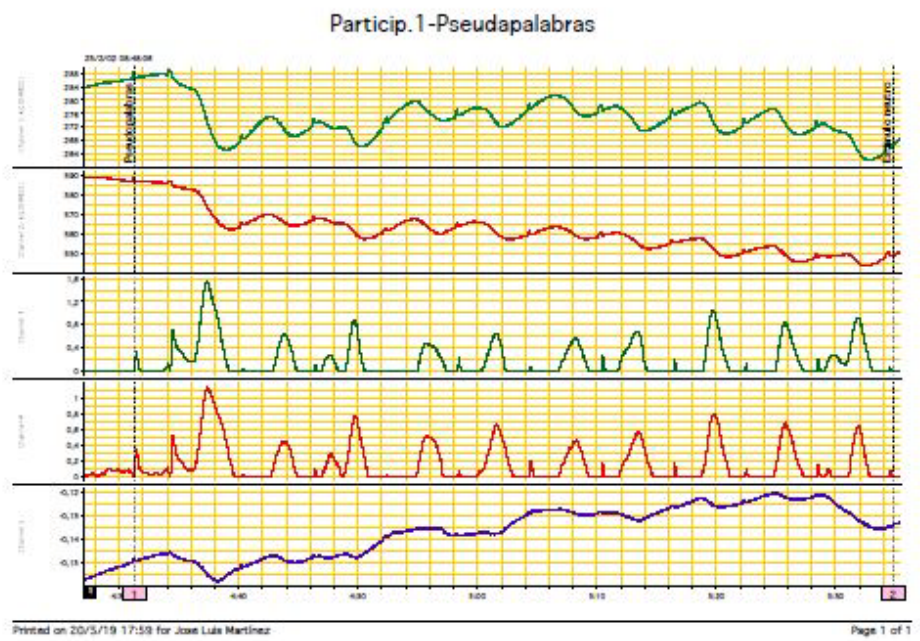

Figura 4. Registro gráfico de la tarea 3 (pseudopalabras) del participante 1 


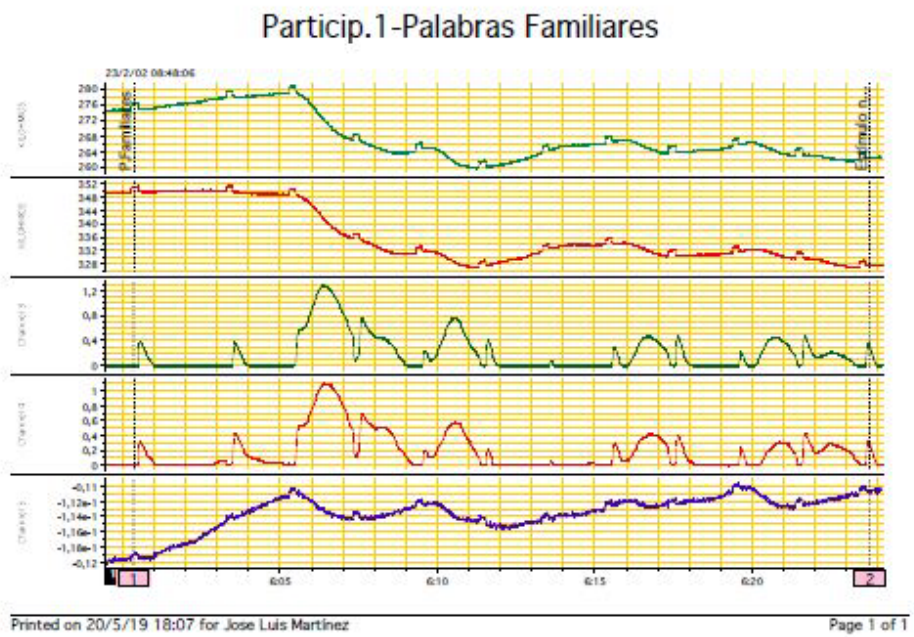

Figura 5. Registro gráfico de la tarea 4 (palabras familiares) del participante 1

En cada uno de los registros, los canales utilizados fueron: 1) Canal 1 (verde), corresponde a la actividad EDL.HD, nivel de actividad electrodermal del hemisferio derecho; 2) Canal 2 (Rojo), corresponde a la actividad EDL.HI, nivel de actividad electrodermal del hemisferio izquierdo; 3) Canal 3 (verde), corresponde a la actividad EDR.HD, respuesta electrodermal del hemisferio derecho; 4) Canal rojo, corresponde a la actividad EDR.HI, respuesta electrodermal del hemisferio izquierdo; y 5) Canal 5 (morado), corresponde a la variable Asimetría, que informa de la dirección de los cambios.

En cada uno de ellos se observan los tiempos en la base del registro, los marcadores que indican el inicio de la presentación de cada una de las palabras en las distintas tareas y, en los canales 3 y 4 , las reacciones a cada en tiempo real. También pueden observarse amplitudes distintas en función de la dificultad de la tarea.

Cada uno de los canales se describe con escalas diferentes, reflejadas en la parte izquierda de cada registro. Para presentarlos se utilizan autoescalas, ajustadas a los valores máximos y mínimos de cada variable, para una mejor representación gráfica.

Podemos observar en las tareas 1 (euskera) y 2 (sueco) que las respuestas son muy evidentes y pronunciadas, mostrando una activación hacia el hemisferio derecho. Sin embargo, los registros de las tareas 3 (pseudopalabras) y 4 (familiares) presentan cambios de menor rango. Finalmente, en la tarea 4 (familiares), el canal 5, Asimetría, aparece sin apenas variaciones.

\section{Análisis cuantitativo del estudio 1}

Para este se presentan, en primer lugar, los datos obtenidos en los registros. Puesto que estos son muy numerosos, nos centraremos solo en los más relevantes. 


\section{PATRÓN DE ACTIVACIÓN HEMISFÉRICA EN ADULTOS EN TAREAS DE LECTURA DE PALABRAS Y CONCIENCIA FONOLÓGICA MEDIDAS CON UNA NUEVA METODOLOGíA DE MEDIDA DE LA ACTIVIDAD ELECTRODERMAL DIFERENCIAL (EDA-D)}

Tabla 2. Datos de los valores de la EDL.HD y EDL.HI y Asimetría (AS.)

\begin{tabular}{llllr} 
Nivel basal & Euskera & Sueco & $\begin{array}{l}\text { Pseudopalabr } \\
\text { as }\end{array}$ & Familiares \\
HD $-439,31$ & 455,18 & 445,59 & $442,51(3,39)$ & 439,74 \\
$(22,53)$ & $(7,01)$ & $(4,47)$ & & $(2,72)$ \\
HI $-451,86$ & 466,75 & 459,39 & $456,92(3,30)$ & 453,39 \\
$(21,34)$ & $(6,78)$ & $(4,61)$ & & $(2,90)$ \\
AS.,- 01715 &,- 01438 &,- 02229 &,- 01873 &,- 01821 \\
\hline
\end{tabular}

Los valores HD y HI corresponden a $\mathrm{K} \Omega$ (desviación entre paréntesis). Los valores de AS. son porcentajes de desviación respecto al valor 0 . Puesto que todos los valores son negativos el grupo, en conjunto, muestra una activación hemisférica basal derecha $(60 \%)$.

La tabla 2 recoge los valores basales y los de las cuatro tareas. Estos datos, analizados por género, no presentan diferencias significativas en las tres variables registradas. La variable asimetría presenta algunas diferencias que se explicarán posteriormente.

Para comparar los datos de estas variables entre cada una de las tareas recurrimos a la formulación de Spearman para la comparación de muestras que no tienen medias ni desviaciones iguales. Para ello, utilizamos el Coeficiente de Variación de Spearman (CV), con variables transformadas para cada tarea, denominadas:

$\circledR$ Tarea 1 - palabras en euskera

T1-CV-HD y T1-CV-HI
T2-CV-HD y T2-CV-HI
T3-CV-HD y T3-CV-HI
T4-CV-HD y T4-CV-HI

${ }^{\circledR}$ Tarea 2 - palabras suecas

@ Tarea 3 - pseudopalabras

( T) Tarea 4 - palabras familiares

La Tabla 3 ofrece los valores obtenidos de los Coeficientes de Variación de las cuatro tareas, ya transformadas.

Tabla 3- Valores de las medias de los coeficientes de variación de las tareas de ambos hemisferios

$\begin{array}{llll}\text { T1- Euskera } & \text { T2- Sueco } & \begin{array}{l}\text { T3- } \\ \text { Pseudopalabras }\end{array} & \text { T4 - Familiares } \\ & & \end{array}$

\begin{tabular}{lrrrr}
\hline CV-HD & 1,5493 &, 9842 &, 7940 &, 6653 \\
CV-HI & 1,4752 &, 9832 &, 7304 &, 6618
\end{tabular}

Los datos obtenidos de CV-HD y CV-HI se han comparado por tareas mediante la prueba $T$ de Student para muestras relacionadas, no existiendo diferencias significativas entre ambos hemisferios en ninguna de las cuatro. A su vez, se realizó el mismo análisis para comparar los datos entre cada una de las tareas. Las Tablas 4 y 5 presentan dichos datos separadamente para los valores CVHD y CV-HI. 
Tabla 4- Resultados prueba T de Student para muestras relacionadas de los valores CV- HD

\begin{tabular}{lrcc}
\hline Pares relacionados & Valor $\boldsymbol{t}$ & Significación \\
\hline T1-CV-HD (Euskera) & T2-CV-HD (Sueco) & 3,791 &, 001 \\
T1-CV-HD (Euskera) & $\begin{array}{c}\text { T3-CV-HD } \\
\text { (Pseudopalabras) }\end{array}$ & 4,263 &, 000 \\
T1-CV-HD (Euskera) & T4-CV-HD (Familiares) & 5,410 &, 000 \\
T2-CV-HD (Sueco) & $\begin{array}{c}\text { T3-CV-HD } \\
\text { (Pseudopalabras) }\end{array}$ & 1,850 &, 074 \\
T2-CV-HD ( Sueco) & T4-CV-HD (Familiares) & 2,882 &, 007 \\
$\begin{array}{l}\text { T3-CV-HD } \\
\text { (Pseudopalabras) }\end{array}$ & T4-CV-HD (Familiares) & 1,405 &, 177 \\
\hline
\end{tabular}

$\mathrm{gl}=29$

Tabla 5- Resultados prueba T para muestras relacionadas de valores de CV-HI

\begin{tabular}{llcc}
\hline Pares relacionados & & Valor t & Significación \\
\hline T1-CV-HI (Euskera) & T2-CV-HI (Sueco) & 2,569 &, 016 \\
T1-CV-HI (Euskera) & $\begin{array}{l}\text { T3-CV-HI } \\
\text { (Pseudopalabras) }\end{array}$ & 3,652 &, 001 \\
T1-CV-HI ( Euskera) & T4-CV-HI (Familiares) & 4,177 &, 000 \\
T2 -CV-HI (Sueco) & $\begin{array}{l}\text { T3-CV-HI } \\
\text { (Pseudopalabras) }\end{array}$ & 3,314 &, 002 \\
T2-CV-HI (Sueco) & T4-CV-HI (Familiares) & 3,683 &, 001 \\
$\begin{array}{l}\text { T3-CV-HI } \\
\text { (Pseudopalabras) }\end{array}$ & T4-CV-HI (Familiares) & 1,135 &, 266 \\
\hline
\end{tabular}

$\mathrm{gl}=29$

Los valores de $t$ y sus niveles de significación indican diferentes niveles de activación en cada tarea. Aparecen diferencias significativas entre la tarea 1 (euskera) y la 2 (sueco), tanto en el hemisferio derecho como en el izquierdo, siendo mayor la diferencia en el derecho (HD=3,791; $\mathrm{HI}=$ 2,569). El mismo fenómeno ocurre al comparar la tarea 1 (euskera) con la 3 (pseudopalabras), con diferencias muy significativas, siendo más acusado este efecto en el $\mathrm{HD}(\mathrm{HD}=4,263 ; \mathrm{HI}=3,652)$. Al comparar la tarea 1 (euskera) y la 4, (familiares) también aparecen diferencias significativas, mayores en el $\mathrm{HD}(\mathrm{HD}=5,410 ; \mathrm{HI}=4,177)$. Al comparar la tarea 2 (sueco) y la 3 (pseudopalabras), se reflejan diferencias significativas, y entre la tarea 2 (sueco) y la 4 (familiares). En estos dos últimos 


\section{PATRÓN DE ACTIVACIÓN HEMISFÉRICA EN ADULTOS EN TAREAS DE LECTURA DE PALABRAS Y CONCIENCIA FONOLÓGICA MEDIDAS CON UNA NUEVA METODOLOGíA DE MEDIDA DE LA ACTIVIDAD ELECTRODERMAL DIFERENCIAL (EDA-D)}

casos, observamos que los mayores valores de $t$ corresponden, a diferencia de lo encontrado anteriormente, a una mayor activación izquierda. Al relacionar la tarea 2 (sueco) con la 4, (familiares), los valores de $t$ son más elevados en el $\mathrm{HI}(\mathrm{HI}=3,683 ; \mathrm{HD}=2,882)$. En la relación entre la tarea 3 (pseudopalabras) y la 4 (familiares) no aparecen diferencias significativas.

Si analizamos las desviaciones estándar en cada tarea, consideraríamos que las respuestas de los participantes serían más o menos homogéneas o divergentes en función de su dificultad. Observamos que es la tarea $\mathrm{T} 1$ (euskera) la que presenta mayor $\mathrm{SD}(\mathrm{HD}=7,01 ; \mathrm{HI}=6,78$ ), le sigue la T2 con una desviación menor ( $\mathrm{HD}=4,47 ; \mathrm{HI}=4,61)$, más alta que en la T4 (familiares) con valores de SD muy bajos y homogéneos ( $\mathrm{HD}=2,72 ; \mathrm{HI}=2,90)$. Observando, además, algunas diferencias en la activación diferencial.

En la variable Asimetría, aparecen diferencias significativas en cada una de las cuatro tareas, aunque los valores indican que es en la tarea 2 (sueco), donde existe una mayor una mayor activación del hemisferio derecho. En la prueba de $t$, al comparar la activación hemisférica entre la tarea 1 (euskera) con la 2 (sueco), se obtiene una $t=1,530 ; p, 137$. No se logra, por tanto, un valor de significación pero los datos muestran una tendencia hacia una mayor activación derecha en esta al compararla con la 1.

\section{Análisis cuantitativo del estudio 2}

En la tabla 6 se presentan los datos de las variables básicas. En ella se incluye la variable Slope 0 pendiente.

Tabla 6. Datos de los valores de la EDL.HD y EDL.HI y Asimetría en las tareas T5 y T6

\begin{tabular}{llll}
\hline & Nivel basal & \multicolumn{1}{c}{ T5- Primer sonido } & $\begin{array}{c}\text { T6- Tercer } \\
\text { sonido }\end{array}$ \\
EDL-HD & $439,31(22,53)$ & $419,53(4,27)$ & $418,90(4,07)$ \\
EDL-HI & $451,86(21,34)$ & $433,12(3,71)$ & $432,72(3,59)$ \\
Slope EDL- &,- 04184 &, 03034 \\
HD & &, 02714 &, 04031 \\
$\begin{array}{l}\text { Slope EDL- } \\
\text { HI }\end{array}$ & &,- 01740 &,- 01961 \\
\hline Asimetría &,- 01715 &
\end{tabular}

Los valores $\mathrm{HD}$ y $\mathrm{HI}$ corresponden a $\mathrm{K} \Omega$. El paréntesis indica la desviación. Los valores de Slope positivos, indican aumento de los niveles de resistencia, los negativos, disminución.

En la tabla 7 se presentan las variables HD y HI transformadas al Coeficiente de Variación. 
PSICOLOGÍA POSITIVA

Tabla 7- Valores de las medias de los Coeficientes de Variación (CV) de las tareas en ambos hemisferios

\begin{tabular}{llc}
\hline & & Valores de CV \\
\hline $\begin{array}{l}\text { T5 - CV- } \\
\text { HD- }\end{array}$ & Tarea primer Sonido & 1,0381 \\
$\begin{array}{l}\text { T5 - CV- } \\
\text { HI- }\end{array}$ & Tarea Primer sonido &, 8591 \\
$\begin{array}{l}\text { T6- CV- } \\
\text { HD- }\end{array}$ & Tarea tercer sonido &, 9772 \\
$\begin{array}{l}\text { T6- CV- } \\
\text { HI- }\end{array}$ & Tarea tercer sonido &, 8146 \\
\hline
\end{tabular}

Analizamos si los datos de CV-HD y CV-HI en ambas tareas presentan diferencias significativas mediante una $t$ de Student para muestras relacionadas. Los datos refieren que los valores de T5-CVHD y T5-CV-HI no difieren significativamente entre si $(t=1,266 . \mathrm{gl}, .29 ; p=, 216)$, pero en la T6, Ios valores de T6-CV-HD y T6-CV-HI difieren significativamente ( $t=2,111 . g l, 29 ; p=, 043)$. Teniendo en cuenta esto, aparece una significativa diferencia de activación diferencial, atribuible a una mayor variabilidad en la resolución de la tarea, ya que las varianzas de la T5 son mayores que las de la T6.

En la variable Asimetría, observamos que en la T5, el valor indica una participación del hemisferio derecho, pero en la tarea 6 , la tendencia aparece más acusada ( $t=-, 498, g l .2 ;, p=, 622)$.

En ambas se incluyó el análisis de la pendiente o Slope. La prueba de $t$ indica que en la T5 aparece una diferencia significativa entre estas variables Slope T5-EDL-HD y Slope T5- EDL-HI ( $t=-$ 2,201 gl .29; $p=, 036$ ). Ello supone, al ser negativa y significativa, una mayor activación del hemisferio derecho sobre el izquierdo.

\section{DISCUSIÓN Y CONCLUSIONES}

Nuestra pretensión ha sido comprobar si el instrumento y la metodología propuesta permitía identificar patrones de activación hemisférica en tareas de lectura de palabras y conciencia fonológica. A partir de los resultados obtenidos hemos identificado diferencias significativas en la activación hemisférica entre las tareas analizadas.

En el estudio 1, comprobamos que al comparar T1 (euskera) con T2 (Sueco), T3 (pseudopalabras) y T4 (familiares) se observan diferencias significativas en la activación hemisférica tanto en el HI como en el HD, aunque en este ultimo más acusadas. Por el contrario, al comparar la T2 (Sueco) con la T3 (pseudopalabras) y la T4 (familiares) se observa que la mayor activación se produce en el $\mathrm{HI}$. Es posible que para ejecutar ambas tareas (T3 y T4) se active de forma preferente el HI por dos motivos: 1) en pseudopalabras, por su escasa imaginabilidad y 2) en familiares, debido a que al ser lectores expertos son leídas por ruta léxica (Porta, Kraft y Harper, 2009).

Por otro lado, no aparecen diferencias entre la T3 (pseudopalabras) y la T4 (familiares), dato coherente con los estudios que atribuyen las dos vías de acceso al léxico al hemisferio izquierdo (Dehaene, 2018), sin embargo, no es así en la tarea de lectura de palabras suecas, es posible que en este proceso se utilicen estructuras de decodificación fonológica diferentes ya que la estructura silábica sueca es muy consonántica y puede obligar a recurrir a elementos diferentes a la fonología, como la prosodia; no obstante, este dato sería necesario confirmarlo con una replicación del estudio y validarlo con una muestra más amplia. En la variable asimetría aparece una mayor activación 


\section{PATRÓN DE ACTIVACIÓN HEMISFÉRICA EN ADULTOS EN TAREAS DE LECTURA DE PALABRAS Y CONCIENCIA FONOLÓGICA MEDIDAS CON UNA NUEVA METODOLOGÍA DE MEDIDA DE LA ACTIVIDAD ELECTRODERMAL DIFERENCIAL (EDA-D)}

del HD en la T2 (sueco) y una tendencia también hacia el derecho al comparar la T1 (euskera) con la T2 (sueco). Estos datos, de nuevo, podrían ser explicados por la singular estructura consonántica de las palabras suecas.

El hecho de que exista una mayor activación en el HI en T1 (euskera), T3 (pseudopalabras) y T4 (familiares) podría ser explicado por la transferencia de los procesos implicados en la decodificación fonológica entre distintas lenguas que comparten un mismo código (Ferrero y Goikoetxea, 2007).

En el estudio 2 no se han obtenido resultados tan evidentes. En la T5 (primer sonido) no aparecen diferencias significativas en la activación diferencial, aunque sí se observa una mayor tendencia hacia el HD en las variables Slope y Asimetría. Es posible que primero se active el HI para realizar la tarea de conciencia fonológica (Mejía y Eslava, 2008) y, después, se recurra a la prosodia para lograr producir la palabra (HD) de este modo, se justificaría la participación de ambos hemisferios y se podrían explicar los resultados de las variables Slope y Asimetría. Se observó también una mayor varianza, probablemente debida al bajo porcentaje de aciertos de los participantes (26,6\%) en la tarea y, en consecuencia, a las distintas estrategias para resolverla.

Al comparar la activación derecha e izquierda en la T6 (tercer sonido), aparecen diferencias significativas, aunque no podemos ofrecer una explicación clara de estos resultados debido a la dificultad de interpretación del Coeficiente de Variación.

Por otro lado, los datos de medida de la actividad basal del grupo refieren una activación hemisférica derecha en la mayor parte de los participantes (60\%), esto puede haber condicionado de algún modo los resultados obtenidos en las tareas, cuestión que lleva a plantearse si estos hubieran sido los mismos en el caso de que la muestra hubiera estado compensada.

Este estudio, aunque preliminar, puede abrir un campo nuevo y un yacimiento de ideas en la compleja tarea de comprender mejor algunos procesos cognitivos como los que aquí se presentan, para ello, sería necesario introducir elementos de mejora metodológica, una replicación del estudio con una muestra más amplia, y un análisis más complejo de las variables utilizadas.

\section{REFERENCIAS BIBLIOGRÁFICAS}

Alegría, J., Carrillo, M., \& Sánchez, E. (2005). La enseñanza de la lectura. Investigación y Ciencia, 340 (1), 6-14.

Ardila, A., Bernal, B., y Rosselli, M. (2016). The language area of the brain: a functional reassessment. Revista de Neurologia, 62 (3), 97-106.

Boucsein, W. (2012). Electrodermal activity. London: Springer.

Cantú, D., Lera, J. A., y Baca, J. R. (2017). Especialización hemisférica y estudios sobre lateralidad. Revista de psicología y ciencias del comportamiento de la Unidad Académica de Ciencias Jurídicas y Sociales, 8(2), 6-50.

Colheart, M. (1978). Lexical access in simple reading task. Strategies in information processing, 151-216.

Cuetos, F. (2018). Psicología de la lectura. Madrid: Panamericana.

Cuetos, F., Rodríguez, B., Ruano, E. y Arribas, D. (2007). Prolec-R. Evaluación de los procesos lectores-revisado. Madrid: TEA.

Defior., Serrano, F. y Gutiérrez, N. (20015). Dificultades específicas de aprendizaje.

Síntesis.

Dehaene, S. (2018). El cerebro lector. Madrid: Siglo XXI.

Fajardo, A., Hernández, J., y González, Á. (2012). Acceso léxico y comprensión lectora: un estudio con jóvenes universitarios. Revista electrónica de investigación educativa, 14(2), 25-33. 
Fawcett, A., y Nicolson, (2013). DST-J: test para la detección de la dislexia en niños.

Madrid:TEA.

Ferrero, M., y Goikoetxea, E. (2007). Entrenamiento en conocimiento fonológico en lengua euskera: estudio de caso único. Revista de Logopedia, Foniatría y Audiología, 27(4), 198-203.

Freixa i Baqué, E. (1984). Asymmetry electrodermal activity: a review. Biological Psychology, 18, 219-239.

Gerrits, R., Van der Haegen, L., Bryshaert, M., y Vingerhoets, G. (2019). Laterality for Recognizing Written Words and Faces in the Fusiform Gyrus covaries with Language Dominance. Cortex,117, 196-204.

Hellerud, B.C. (2002). Skin conductance and behaviour during sensory stimulation of preterm and term infants. Early Human Development, 70, 1/2, 35-47.

López-Escribano, C. (2009). Aportaciones de la neurociencia al aprendizaje y tratamiento educativo de la lectura. Aula, 15, 47-78.

Martínez, J.L. y Valdunquillo, M.I., (1993). Aspectos evolutivos en el desarrollo de la especialización hemisférica medidos a partir de la actividad electrodérmica diferencial. En J.A. Beltrán, L.Pérez, E. Gonzáles, R.González y D. Vence: Líneas actuales en la intervención psicopedagógica I: Aprendizaje y contenidos del currículum. (pp. 201-214). Madrid: Universidad Complutense de Madrid.

Martínez, J.L., Garrido, E. y Valdunquillo, M.I. (2005). Desarrollo tecnológico para la medida electrofisiológica de la atención y activación emocional en grupos sociales -Sociograph-. En J. Romay-Martínez y R. García-Mira (Eds.), Aspectos históricos, teóricos y metodológicos (pp.216-221). Madrid: Biblioteca Nueva.

Martínez, J. L., Monge, S. y Valdunquillo, M.I. (2012). Medición de las respuestas psicofisiológicas para apoyar el análisis de discursos políticos. Trípodos, 29, 53- 72.

Mejía, L., y Eslava, J. (2008). Conciencia fonológica y aprendizaje lector. Acta Neurológica Colombiana, 24(S2), S55-S63.

Mojica-Londoño, A. G. (2017). Actividad electrodérmica aplicada a la psicología: análisis bibliométrico. Revista Mexicana de Neurociencia, 18(4), 46-56.

Moncada, M.E., y De la Cruz, J. (2011). La actividad electrodérmica- Revisión. Ingeniería e investigación, 31(2), 143-151.

Monsalve, A., y Cuetos, F. (2001). Asimetría hemisférica en el reconocimiento de palabras: efectos de frecuencia e imaginabilidad. Psicothema, 13(1), 24-28.

Porta, M. E., Kraft, R., y Harper, L. (2009). Hemispheric asymmetry profiles during beginning reading: Effects of reading level and word type. Developmental Neuropsychology, 35(1), 96-114.

Ramos, J., y Cuadrado, I. (2006). Prueba para la Evaluación del Conocimiento Fonológico - PECO. Madrid: EOS.

Rippon, G. (1993). Individual differences in electrodermal and EEG asymmetries.

International Journal of Psychophysiology. 8, 3, 309-320.

Roesmann, K., Dellert, T., Junghoefer, M., Kissler, J., Zwitserlood, P., Zwanger, P. y Dobel, Ch. (2019). The causal role of prefrontal hemispheric asymmety in valence processing of words -Insights from a combined cTBS-MEG study. Neuroimage, 367-379.

Román-Lapuente, F. García-Sánchez, F.A. y Gómez-Amor, J. (1986). Principales medidas de asimetría hemisférica. Anales de Psicología, 3, 55-66.

Rueda, M.I. (2017). El aprendizaje de la lectura y sus dificultades. En S.D. Martinelli. y

D.C. Fernández. (Ed.), Aprendizagem escolar na contemporaneidade (pp. 89- 116). Curitiba, Brasil: Juriá. 
